\title{
The anti-oxidant capacity of green tea polyphenols in the oral cavity
}

\author{
M. Asim ${ }^{1}$, O. Han Liu ${ }^{1}$, E. Okello ${ }^{1}$, M. Birch-Machin ${ }^{2}$ and M. Foltz ${ }^{3}$, G. Lietz ${ }^{1}$ \\ ${ }^{1}$ Human Nutrition Research Centre, Agriculture Building, Newcastle University, Newcastle Upon Tyne NE1 7RU, UK, \\ ${ }^{2}$ Institute of Cellular Medicine, Newcastle University, Newcastle Upon Tyne NE1 7RU, UK and ${ }^{3}$ Molecular Aspects of \\ Health, Unilever Research \& Development, Vlaardingen, The Netherlands
}

Green tea polyphenols have attracted considerable attention in the past 20 years as potential therapeutics in the prevention of cancers, cardiovascular diseases, and neurodegenerative diseases ${ }^{(1)}$. However, the bioavailability of tea polyphenols is limited by their extensive biotransformation through phase II enzymes (COMT, UGTs, and SULTs) after ingestion \& absorption ${ }^{(2)}$.

To test the antioxidant capacity of green tea in the oral cavity, we recruited nine volunteers and extracted their buccal mucosal cells before and after exposure to green tea at different timepoints. Reactive oxygen species (ROS) concentration was measured using the DCFH-DA assay, and absorption of catechins in the oral mucosa was determined by HPLC. The results showed significantly reduced ROS levels when comparing baseline to tea treated cells, with maximum reduction of ROS concentrations within 10 minutes of exposure. HPLC analysis indicated that only EGCG and ECG were absorbed by buccal mucosal cells (Figure 1).
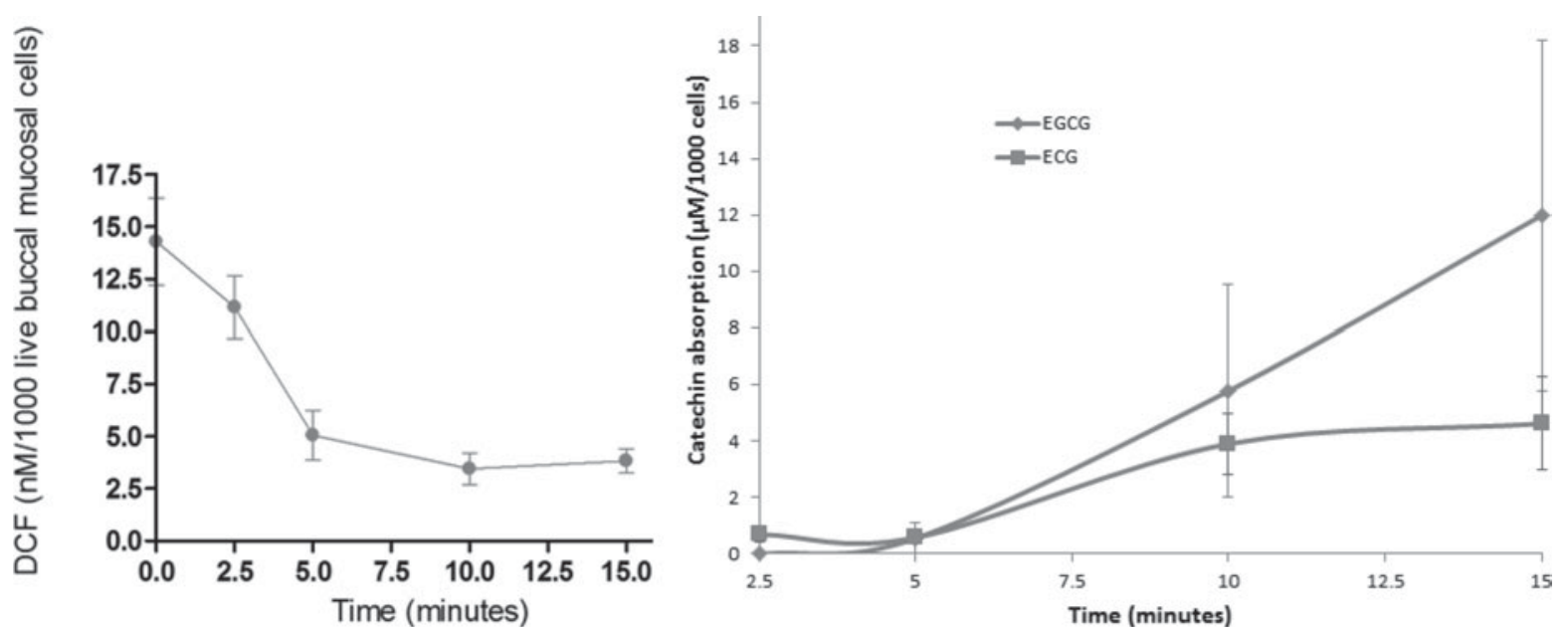

Fig. 1. ROS concentrations in buccal mucosal cells depending on exposure to green tea polyphenols (left) and absorption of catechins in buccal cell extracts (right).

In summary, EGCG and ECG are absorbed in the oral cavity and reduce ROS concentrations after only 2.5 minutes of incubation. The study also observed large inter-individual variations in absorption of catechins and their associated antioxidant capacity which warrants further investigation.

1. Katiyar S \& Mukhtar H (1996), Int J Oncol,. 8(2), 221-38.

2. Hollman PC, Tijburg LB \& Yang CS (1997) Crit Rev Food Sci Nutr. 37(8), 719-38. 